the health service that tells doctors which hospital and consultant can see their patients most quickly. Has outside industry anything to teach the health service? For instance, could the system be organised like the travel business so that customers (patients) are linked to flights (theatre sessions) and hotels (hospitals) to provide holidays (treatment)? As part of the national waiting list initiative North West Thames Regional Health Authority and North Hertfordshire District Health Authority are jointly piloting an information service that will explore and identify the sort of information that will help general practitioners to obtain earlier treatment for their patients.

Enthoven proposed an "internal market model" to solve the present ills of the NHS.${ }^{34} \mathrm{He}$ visualised that an authority would provide and pay for the health care of its residents but be able to obtain further payments at a fixed rate for the emergency care of non-residents and to negotiate an even higher fee for a non-emergency service. This conjures up the possibility of health authorities competing with one another and perhaps the private sector to provide certain forms of health care; and patients would travel from one end of Britain to another for treatment. This has already begun. Gloucester Health Authority recently contracted with Victoria (now Riverside) Health Authority to provide total hip replacement operations at $£ 1200$ a hip, and several authorities have contractual arrangements with the private sector. ${ }^{5}$ There is also an opportunity to test some ideas. The projects funded by the national waiting list initiative will allow authorities to compare and contrast the cost, treatment, and care provided for similar cases by the private sector, by locally expanded NHS facilities, and by other health authorities using their spare capacity.

Health care is provided free at the time of use, and waiting lists are a way of controlling the access that patients have to treatment. In other countries, such as the United States, the income of the patient is the rationing mechanism. Waiting lists are misunderstood by both doctors and the public. Doctors have used them to show a need for resources and to manage patients whose illness will improve with time; politicians and the public view them as the measure of government performance on the delivery of health care. Yet waiting lists for treatment are put together in many different ways. They are subject to the working practices of particular consultants, whose systems may be quite different from those of their colleagues in the same specialty as well as from those in other specialties. The data collection systems are just as inconsistent. ${ }^{2}$

Some health authorities have tried to shorten their waiting lists by getting agreement among doctors, ${ }^{26}$ by having common waiting lists and preassessment clinics, by encouraging weekend operating sessions, and by providing managers to organise and coordinate outpatient attendances, admissions, and the number of beds and theatre sessions in a specialty.$^{78}$ Others believe that waiting times would shorten if doctors were more efficient, adopted new techniques, and increased their throughput of patients. This they might do by increasing the numbers of day and short stay cases and the amount done in outpatient clinics. ${ }^{2}$ The Royal College of Surgeons of England suggests that the amount of day care could be increased,' but national and professional edicts require local will and facilities (including adequate retraining) to happen.

The national care system does require more resources to provide better facilities and modern equipment and to maintain adequate numbers of nurses, technicians, and junior doctors. It also needs scope to test and explore some of these new ideas. There is a risk that district health authorities and hospitals will give higher priority to non-emergency patients for whom they receive additional payment than to local residents and that the patients who live further away will be less likely to be referred.

After almost 40 years of the same referral systems and waiting practices, is it not time for consultants, general practitioners, and managers to test out alternative ways to provide health care free at time of use for their patients?

MAUREen Dalziel

Specialist in Community Medicine,

North West Thames Regional Health Authority,

London WC2 3QR

District General Manager,

North Hertfordshire Health Authority,

Hitchin SG5 $1 \mathrm{HE}$

1 MacArthur C, Pendelton LL, Smith A. Treatment delay in patients with bladder tumours. f Epidemiol Community Health 1985;39:63-6.

2 Department of Health and Social Security. Waiting for hospital treatment. London: Department of Health and Social Security, 1980.

3 Enthoven AC. Reflections on the management of the National Health Service. London: Nuffield Provincial Hospitals Trust, 1985.

4 Anonymous. Gridlock and incentives in NHS. Br Med $\mathcal{Y}$ 1985;291:992-3.

5 Aaing W. Private health care. London: Office of Health Economics, 1985.

6 Porter KM. Orthopaedic audit-review of inpatient waiting lists. Br Med $\mathcal{F}$ 1985;291: 1216-7.

7 Pierce A. New post for an old problem. Health and Social Services fournal 1985;94:420-2.

8 Heppenstall I. Entering the twilight zone. Health and Social Services foumal 1985;94:982.

9 Royal College of Surgeons of England. Guidelines for day care surgery. Commission on the provision of surgical services. London: Royal College of Surgeons of England, 1985.

\section{Overuse of monitoring of blood concentrations of antiepileptic drugs}

Monitoring serum or plasma concentrations of antiepileptic drugs has become routine for largely historical reasons: phenytoin has a non-linear relation between the dose and the serum concentration ${ }^{1}$ and a dose related neurotoxicity (drowsiness, ataxia, dysarthria, and nystagmus). This results in a narrow therapeutic window, and monitoring is necessary to avoid neurotoxicity in patients who continue to have seizures. The concept of the "therapeutic" or "optimal" range for phenytoin has been extended to other antiepileptic drugs, and many laboratories now routinely estimate serum concentrations of drugs other than phenytoin. This is a questionable practice.

A single measurement will give a good approximation of the steady state for drugs with long half lives (phenytoin and phenobarbitone) but not for drugs with short half lives. Measurements of sodium valproate concentrations from specimens taken at random during the day are virtually uninterpretable as they may represent unpredictable peak, trough, or intermediate concentrations. ${ }^{2}$ Collecting early morning specimens for measuring troughs is, however, rarely practicable.

Doctors must be aware of what is measured during routine estimations of blood concentrations of antiepileptic drugs and, perhaps more importantly, what is not measured. Some drugs have metabolites that seem to contribute to the therapeutic effect but which are not routinely monitored. These include the 10,11-epoxide of carbamazepine ${ }^{3}$ and phenylethylamonamide derived from primidone. ${ }^{4}$ Most laboratories determine the drug concentration in whole 
plasma or serum. Phenytoin, carbamazepine, and sodium valproate are heavily protein bound, but only the free drug fraction is in equilibrium with the brain and pharmacologically active. Though variation in protein binding of drugs is usually not clinically important, upsets in the relation may occur in hepatic and renal failure ${ }^{5}$ and pregnancy and because of drug interaction-for example, phenytoin with sodium valproate. The salivary concentrations of phenytoin and carbamazepine correlate well with free drug concentrations, but this is not so for sodium valproate. Salivary measurements may thus be more meaningful, but they are not used by many laboratories. Measurement of free drug concentrations by equilibrium dialysis or ultrafiltration techniques are expensive and not readily available.

Even when concentrations of free drugs and their metabolites in blood are known important pharmacodynamic considerations may alter the relation between the blood concentration and the therapeutic effect. Thus for sodium valproate the onset of action is slower and longer lasting than can be explained by the pharmacokinetics of the drug. ${ }^{6}$ Similarly tolerance to the neurotoxicity and therapeutic effects of benzodiazepines and barbiturate drugs must be caused by unexplained changes in drug-receptor interaction.

There are further fundamental biological reasons for doubting the value of routine monitoring of blood concentrations of antiepileptic drugs. The upper limit of a therapeutic range may be defined as the concentration of the drug at which toxic effects are likely to appear. The most consistent relation between the serum concentration and toxic effect is for phenytoin, but even with this drug some patients may tolerate and indeed require serum concentrations above $20 \mu \mathrm{g} / \mathrm{ml} .^{7}$ For sodium valproate, phenobarbitone, and carbamazepine there is a wide variation in individual tolerance of serum concentrations.

The lower limit of the therapeutic range is even more difficult to define, and many patients have epilepsy that is controlled by anticonvulsant serum concentrations well below the optimal range. ${ }^{89}$ Even for one patient the threshold for suppressing tonic clonic seizures may differ from that for suppressing partial seizures. ${ }^{10}$ Unquestioning acceptance of therapeutic ranges creates problems: patients with satisfactory control of seizures and low blood concentrations of drugs may have their doses needlessly increased, and patients who tolerate and need high blood concentrations may have their doses reduced. Treating patients is much more important than treating blood concentrations.

Monitoring blood concentrations of anticonvulsants remains important in clinical trials of antiepileptic drugs, but routine monitoring should be restricted to certain categories of patients: firstly, those receiving phenytoin or multiple drug treatment in whom dosage adjustment is necessary because of dose related toxicity or poor seizure control; secondly, mentally retarded patients in whom the assessment of toxicity may be difficult; thirdly, patients with renal or hepatic disease and perhaps pregnant patients, ${ }^{11}$ in whom monitoring of free drug concentrations may be indicated; and, finally, patients who may not be complying with treatment.

Consultant Neurologist,

Walton Hospital,

Liverpool L9 1AE

1 Richens A, Dunlop A. Serum phenytoin levels in the management of epilepsy. Lancet 1975;ii: 247-9.

2 Louiseau P, Cenraud B, Levy RH, et al. Diurnal variations in steady-state plasma concentrations of valproic acid in epileptic patients. Clin Pharmacokinet 1982;7:544-52.
3 Faigle JW, Feldmann KF, Baltzer V. Anticonvulsant effect of carbamazepine. An attempt to distinguish between the potency of the parent drug and its epoxide metabolite. In: GardnerThorpe C, Janz D, Meinardi H, Pippenger CE, eds. Antiepilepric drug monitoring. Avon: Pitman Press, 1977:104-8.

4 Schottelius DD. Primidone biotransformation. In: Woodbury DM, Penry JK, Pippenger CE eds. Antiepileptic drugs. New York: Raven Press, 1982:415-20.

5 Reynolds FJ, Ziroyanis PN, Jones NF, Smith SE. Salivary phenytoin concentrations in epilepsy and in chronic renal failure. Lancet 1976;ii:384-6.

6 Rowan AJ, Binnie CD, Warfield CA, Meinardi H, Meijer JWA. The delayed effect of sodium valproate on the photoconvulsive response in man. Epilepsia 1979;20:61-8.

7 Gannaway DJ, Mawer GE. Serum phenytoin concentrations and clinical response in patients with epilepsy. Br f Clin Pharmacol 1981;12:833-9.

8 Shorvon SD, Chadwick DW, Galbraith AW, Reynolds EH. One drug for epilepsy. Br Med $\mathcal{J}$ 1978;i:474-6.

9 Turnbull DM, Howel D, Rawlins MD, Chadwick DW. Which drug for the adult epileptic patient: phenytoin or valproate? Br Med f 1985;290:815-9.

10 Turnbull DM, Rawlins MD, Weightman D, Chadwick DW. "Therapeutic" serum concentration of phenytoin: the influence of seizure type. $\mathcal{F}$ Neurol Neurosurg Psychiatry 1984;47:231-4.

11 Knott C, Williams CP, Reynolds F. Phenytoin kinetics during pregnancy and the puerperium. Br f Obstet Gynaecol 1986;93:1030-70.

\section{Original pack dispensing}

In Britain a pharmacist either dispenses a medicine in the manufacturer's original pack or, more often, takes the product from a large container and "repackages" it in a smaller one. British pharmaceutical manufacturers are campaigning for dispensing of medicines in original packs to become the norm rather than the exception, a move that would bring Britain into line with virtually all other countries in the European Community.

The trend has already been set, and more and more manufacturers have introduced packs that can be dispensed directly to the patient. Good examples are calendar packs for oral contraceptives and some antihypertensives, strip or foil packs of tablets or capsules, pressurised inhalers for antiasthma medication, and tubes of skin creams or ointments. About $40 \%$ of prescriptions are now dispensed in this way, but, as original pack dispensing has advantages for doctors, pharmacists, and patients, we regret that some $60 \%$ are not. A recent survey by Milpro of some 200 general practitioners showed that most saw advantages in original pack dispensing, while a few were worried about loss of flexibility of dosage.

The Association of the British Pharmaceutical Industry aims at full introduction of original pack dispensing within two to three years. Other organisations - such as the Medicines Commission, the BMA, and the Pharmaceutical Society-support the association, and the Department of Health and Social Security is keen to introduce "tamper evident" packaging after experiences of deliberate contamination of medicines. In practice a "tamper evident" pack can only be the manufacturer's original pack delivered unopened to the patient. Manufacturers, prescribers, and dispensers all support two basic types of pack: a short term treatment pack for seven days' treatment or the normally recommended time for a course of treatment; and a long term pack for one month's treatment.

The advantages of original pack dispensing are many. The identity of the product, batch, and company are preserved, which may have medicolegal importance and also allows more effective recall. The product can be more rapidly identified in cases of accidental overdose. The security and stability of the product are improved, and "tamper evident" and child resistant packs can be developed. Patient compliance may be better and dispensing faster and more efficient. Dispensing errors and mislabelling should also be avoided. 